\title{
Phenotypic characterization of sheep populations in Tahtay Maichew district, Northern Ethiopia
}

\author{
Abebe Hailu ${ }^{a}$, Amine Mustefa ${ }^{*}$, ${ }^{\text {, Tesfalem Aseged }}{ }^{\mathrm{a}}$, Abraham Assefa ${ }^{\mathrm{a}}$, Seble Sinkie ${ }^{\mathrm{a}}$ and \\ Semere Tsewene ${ }^{b}$ \\ ${ }^{a}$ Ethiopian Biodiversity Institute, P.O.Box 30726, Addis Ababa, Ethiopia \\ ${ }^{b}$ Ethiopian Biodiversity Institute, Mekelle Biodiversity Center, P.O.Box 30726, Mekelle, Ethiopia
}

\begin{abstract}
Eighteen quantitative measurements and fourteen qualitative characteristics taken from 306 adult sheep (57 rams and 249 ewes) were used to phenotypically characterize sheep populations of Tahtay Maichew district, Ethiopia. Most traits showed significant variation by agro-ecological zone, sex and age groups with higher values generally recorded for rams as compared to ewes. Middle age group animals displayed highest values for several traits, reflecting the optimal production age. Agro-ecological zone affected ewes more than rams. The highland sheep had shortest height at withers, widest shoulder points and longest hair, indicative of adaptation to their environment. Qualitative characteristics of the studied sheep populations such as tail shape, plain coat color pattern, unpigmented skin, hairy fiber and the absence of horn, toggle, ruff and beard suggest that they constitute a previously undescribed sheep breed. Tan coat color differentiated high and midland sheep from lowland sheep where white and brown colors were dominant. Canon bone length, height at withers and tail length were the three most important morphometric variables used in discriminating the sheep populations. On average $66 \%$ of the animals could be classified into their respective agro-ecological zone. Our data suggest that highland sheep populations are distantly related to lowland sheep, while midland sheep are more closely related to lowland sheep. It can be concluded that breeding programs specific to each agro-ecological zone need to be designed for sustainable utilization and conservation of the studied sheep populations. Furthermore, molecular based studies might allow further characterization of Ethiopian sheep breeds.
\end{abstract}

Keywords: Indigenous breed, Sheep genetic resources, Morphological characterization, Qualitative traits, Tigray region, Ethiopia

Citation: Hailu, A., Mustefa, A., Aseged, T., Assefa, A., Sinkie, S., Tsewene, S. (2020). Phenotypic characterization of sheep populations in Tahtay Maichew district, Northern Ethiopia. Genetic Resources 1 (2), 12-22. doi: 10.46265/genresj.SHBD3744.

(C) Copyright 2020 the Authors.

This is an open access article distributed under the terms of the Creative Commons Attribution License (CC BY 4.0), which permits unrestricted use, distribution, and reproduction in any medium, provided the original author and source are credited.

\section{Introduction}

The Ethiopian livestock sector, which is mainly dominated by indigenous animal genetic resources, contributes significantly to the economy and food security of the country (Central Statistical Agency, 2018), providing livelihood for $37-87 \%$ of the country's population (Central Statistical Agency, 2005). This sector contributes $15-17 \%$ and $35-49 \%$ of the total and agricultural Gross Domestic Product, respectively (Michael et al, 2016). Within the livestock sector, small rumi-

${ }^{*}$ Corresponding author: Amine Mustefa (aminemustefa32@gmail.com) nants, especially sheep, provide a sustainable option for smallholder low input-output production systems. Indigenous sheep genetic resources play a major role in developing countries like Ethiopia, as are better adapted to environments which are harsh, marginal and degraded, have low body weight and excellent grazing skills (Misra and Singh, 2002; Degen, 2007). The indigenous sheep genetic resources account for $99.81 \%$ of the total sheep population in Ethiopia (Central Statistical Agency, 2018).

Conducting phenotypic characterization is a prerequisite for sustainable utilization, conservation and improvement of a breed through designing appropriate sheep breeding programs (FAO, 2012). This will fur- 
ther maximize sustainable food security while minimizing pressure on the environment. Ethiopia, one of the major gateways for domestic sheep to Africa (Devendra and Mcleroy, 1982), is believed to have the largest livestock population in Africa with 31.3 million sheep (Central Statistical Agency, 2018) categorized into 14 traditionally recognized and phenotypically distinct sheep populations ( 9 breeds within 6 breed groups) (Gizaw et al, 2008; EBI, 2016). The 9 sheep breeds of Ethiopia are Simien, Short fat tailed, Washera, Gumz, Horro, Arsi, Bonga, Afar and Black Head Somali (BHS) (Gizaw et al, 2008). In literature, sheep populations of the current study area were generally classified as Sekota traditional population and further categorized under the Short fat tailed breed (Gizaw et al, 2008). However, samples were not taken from nearby areas of the current study areas.

Sekota sheep populations were characterized as short fat tail turned-up at end and fused with main part. The population is medium-sized, predominantly brown or white coat color, few blacks with brown belly. The white animals have finer hair or wooly udder-coat, semi-pendulous or rudimentary ears in Wag Himra and Tigray while predominantly rudimentary in Tekeze valley. Sekota sheep population were reared by Agew, Tigray and Amhara communities (Gizaw et al, 2008).

However, due to the country's high ecological and production system variations, some of the breeds were re-characterized in more recent studies, including Simien sheep (Melaku et al, 2019), Short fat tailed sheep (Hayelom et al, 2014; Bimerow et al, 2011; Getachew et al, 2009), Washera sheep (Mengistie et al, 2010), Arsi sheep (Worku, 2018); Afar sheep (Getachew et al, 2009). In addition to these studies, some work was done in the Tigray region on Abergelle sheep (Tajebe et al, 2011) and Tigray Highland sheep (Gebreyowhens and Tesfay, 2016).

Despite the efforts made to characterize the Ethiopian indigenous sheep genetic resources as mentioned above, they have not yet been exhaustive in covering all regions of the country in general and the Tigray region in particular. They also focused on only a few specifically well-known sheep populations. As a consequence, a high sheep diversity remains unstudied, along with the associated diversified ecology, production systems and ethnic groups. Therefore, there is an urgent need for continued characterization and identification to understand the relationships within and among breeds. Thus, the present study was initiated to cover these gaps and phenotypically describe the indigenous sheep populations of Tahtay Maichew district, Central Tigray zone, Ethiopia.

\section{Materials and methods}

\section{Study Areas}

The study was carried out in the Tahtay Maichew district, which is located in the central zone of Tigray National Regional State (Figure 1). The district covers a total area of $18,618 \mathrm{~km}^{2}$ with estimated livestock
Table 1. Climatic factors and sheep population size of the threeagro-ecological zones in the Tahtay Maichew district of Tigray region, Ethiopia.

\begin{tabular}{llll}
\hline Variables & Highland & Midland & Lowland \\
\hline $\begin{array}{l}\text { Altitude in } \\
\text { meters }\end{array}$ & $>2500$ & $\begin{array}{l}1500- \\
2500\end{array}$ & $<1500$ \\
$\begin{array}{l}\text { Temperature in } \\
\text { o C (mean) }\end{array}$ & 9.9 & 19.9 & 30.3 \\
$\begin{array}{l}\text { Annual rainfall } \\
\text { in mm (mean) }\end{array}$ & $600-700$ & $500-600$ & $400-500$ \\
$\begin{array}{l}\text { Sheep } \\
\text { population size }\end{array}$ & 11,816 & 8,903 & 4,476 \\
\hline
\end{tabular}

population size of 247,907 , consisting 75,707 cattle, 55,517 goats, 110 mules, 6,716 donkeys, 25,195 sheep and 84,102 poultry (Atsbeha et al, 2015). The studied areas were categorized as highland, midland and lowland based on the climatic factors in Table 1 .

\section{Site selection and data collection}

Available background information on the existence of unstudied sheep populations adapted to different agro-ecological zones was captured through short pilot survey and focus group discussions with livestock experts and keepers. Two sites (kebeles) were sampled randomly from each agro-ecological zone (see Table 1). Quantitative and qualitative data were recorded from a total of 306 adult sheep (57 rams and 249 ewes) based on data collection procedures outlined in FAO guidelines (FAO, 2012). Studied animals were carefully handled by trained personnel. Quantitative measurements were taken early in the morning of the day before feeding and watering when the animals were calm and standing in an upright position on flat ground.

Eighteen quantitative measurements were collected: body length $(\mathrm{cm})$, body weight $(\mathrm{kg})$, heart girth $(\mathrm{cm})$, height at withers $(\mathrm{cm})$, chest depth $(\mathrm{cm})$, shoulder point width $(\mathrm{cm})$, subs height $(\mathrm{cm})$ rump length $(\mathrm{cm})$, rump width $(\mathrm{cm})$, tail length $(\mathrm{cm})$, tail width $(\mathrm{cm})$, head length $(\mathrm{cm})$, head width $(\mathrm{cm})$, shin circumference $(\mathrm{cm})$, horn length $(\mathrm{cm})$, hair length $(\mathrm{cm})$, canon bone length $(\mathrm{cm})$, ear length $(\mathrm{cm})$, testis circumference $(\mathrm{cm})$. Fourteen qualitative characteristics were also collected: coat color pattern, coat color, skin color, fiber type, ear orientation, head profile, back profile, rump profile, tail type, tail shape and presence of toggle, horn, beard and ruff were recorded.

\section{Statistical Analysis}

Data were entered and managed using Microsoft Excel@ worksheet. Detection of outliers and testing the normality of the quantitative measurements data was performed using the UNIVARIATE procedure of Statistical Analysis Software (SAS) 9.0 (SAS Institute, 2002). Analysis of data on quantitative measurements and qualitative characteristics was carried out using the General Linear Model (GLM) procedure and the frequency (FREQ) procedure of SAS 9.0 software, 


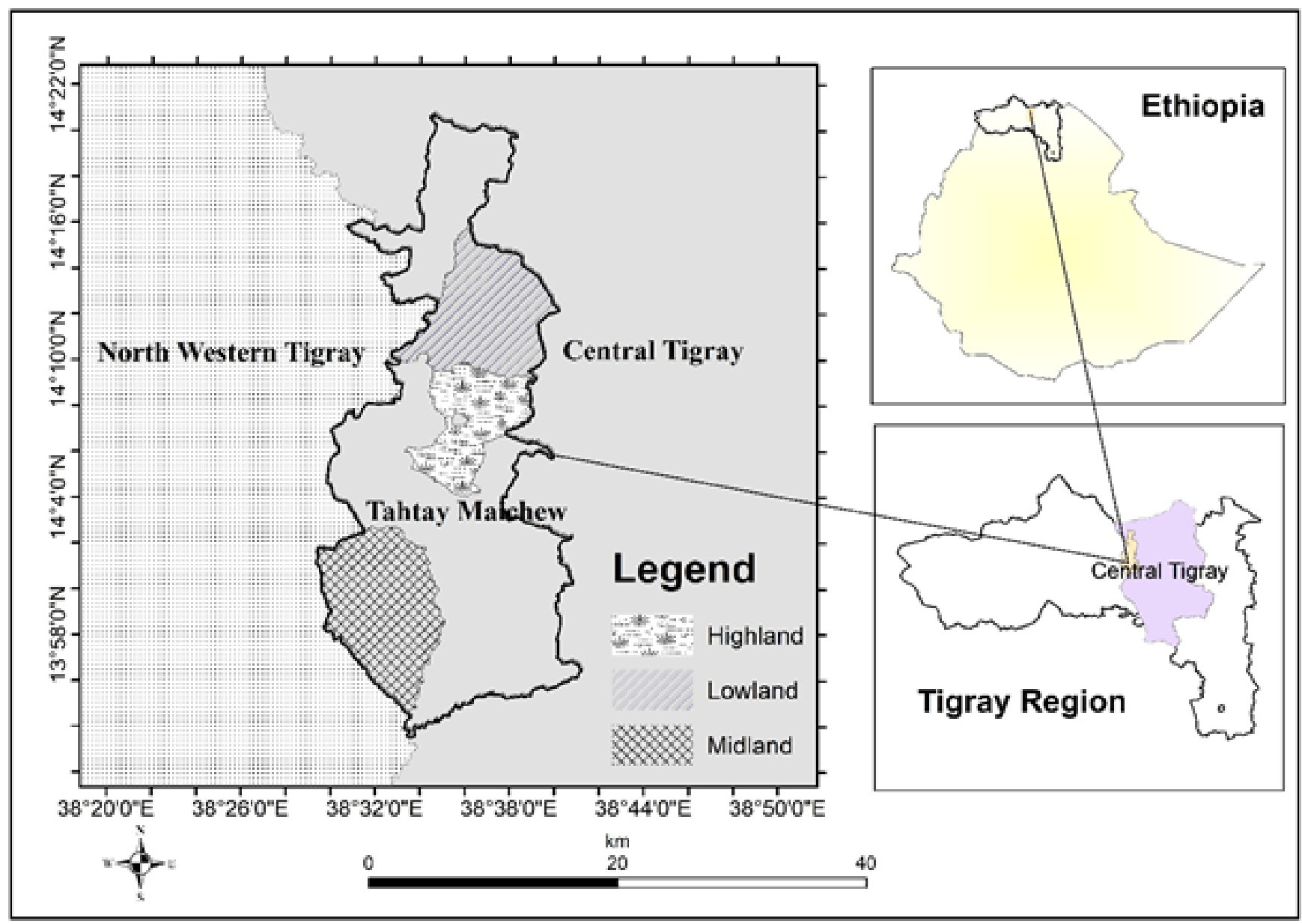

Figure 1. Map of the studied areas.

respectively. Linear measurements Least Square Means (LSM) were separated using the adjusted Tukey-Kramer test (SAS Institute, 2002). Quantitative and qualitative data were analyzed using the following model: $Y_{i j k}=$ $\mu+A_{i}+B_{j}+C_{k}+e_{i j k}$ where $Y_{i j k}$ is an observation, $\mu$ is the overall mean, $A_{i}$ is the fixed effect of environment, $B_{j}$ is the fixed effect of the sex, $C_{k}$ is the fixed effect of age group and $e_{i j k}$ is the random error attributed to the $\mathrm{n}^{\text {th }}$ observation. Environment, sex and age group were fitted as class variables throughout the analysis, while sex effect was removed from the class variables when the analysis was done separately for each sex.

Multivariate analysis was performed on quantitative measurements. Stepwise discriminant function analysis (STEPDISC) with forward selection procedure was used to find out the quantitative variables that better discriminate populations from different environment. Percentage assignment of observations to environment and probabilities of misclassifications were evaluated by discriminant function analysis (DISCRIM). Canonical discriminant function analysis (CANDISC) was also performed to find out linear combination of quantitative variables that provide maximal separations between environments. The scored canonical variables were used to plot pairs of canonical variables to get visual interpretation of environmental differences. Pairwise squared Mahalanobis distances between environments were computed as: $D^{2}(i \mid j)=\left(x_{i}-x_{j}\right)^{\prime} \operatorname{cov}^{-1}\left(x_{i}-x_{j}\right)$. Where $D^{2}(i \mid j)$ is the distances between environments zones $i$ and $j, \operatorname{cov}^{-1}$ is the inverse of the covariance matrix of measured variables, $x_{i}$ and $x_{j}$ are the means of variables in the $i^{\text {th }}$ and $j^{\text {th }}$ populations.

\section{Results}

\section{Quantitative measurements}

Level of significance (P-values) outputs of the class variables for both the overall analysis and separately for each sex are presented in Table 2. Overall, most of the studied traits were significantly affected by agroecological zone, age and sexual differences. Effect of agro-ecological zone on some quantitative traits (heart girth, height at withers, shoulder point width, rump length, tail length, head length, hair length, canon bone length, and ear length) was more significant on ewes than rams.

The overall mean with the respective standard error and deviation, and the effect of agro-ecological zone, sex and age on the quantitative measurements are presented in Tables 3, 4 and 5. The highland sheep population had the shortest height at withers, widest shoulder points and longest hair, while midland sheep population 
Table 2. Level of significance for the overall analysis and separately for both sexes

\begin{tabular}{|c|c|c|c|c|c|c|c|}
\hline \multirow{2}{*}{ Traits } & \multicolumn{3}{|c|}{ Overall } & \multicolumn{2}{|c|}{ Rams } & \multicolumn{2}{|l|}{ Ewes } \\
\hline & Agro-eco zone & Age & Sex & Agro-eco zone & Age & Agro-eco zone & Age \\
\hline $\mathrm{BL}(\mathrm{cm})$ & 0.2688 & $<0.0001$ & 0.3394 & 0.3930 & 0.0028 & 0.3873 & 0.0004 \\
\hline BW (kg) & 0.5309 & $<0.0001$ & 0.9515 & 0.3384 & 0.0061 & 0.6135 & $<0.0001$ \\
\hline $\mathrm{HG}(\mathrm{cm})$ & 0.0330 & $<0.0001$ & 0.6983 & 0.0772 & 0.0002 & 0.0399 & $<0.0001$ \\
\hline HAW (cm) & 0.0005 & 0.0250 & 0.0617 & 0.0562 & 0.0009 & 0.0075 & 0.5451 \\
\hline $\mathrm{CD}(\mathrm{cm})$ & 0.0486 & 0.0013 & 0.0267 & 0.1216 & 0.0120 & 0.2068 & 0.0397 \\
\hline SPW (cm) & 0.0012 & $<0.0001$ & 0.0017 & 0.8511 & 0.0008 & $<0.0001$ & 0.0078 \\
\hline $\mathrm{SH}(\mathrm{cm})$ & 0.5553 & 0.5924 & 0.2194 & 0.1241 & 0.9660 & 0.8881 & 0.7606 \\
\hline $\mathrm{RL}(\mathrm{cm})$ & $<0.0001$ & 0.2364 & 0.2377 & 0.8307 & 0.8157 & $<0.0001$ & 0.1604 \\
\hline $\mathrm{RW}(\mathrm{cm})$ & 0.7008 & 0.0028 & 0.4727 & 0.2884 & 0.0001 & 0.3402 & 0.0504 \\
\hline TL (cm) & 0.0003 & 0.9584 & 0.0040 & 0.1598 & 0.2868 & 0.0007 & 0.2635 \\
\hline TW (cm) & 0.3219 & 0.3538 & $<0.0001$ & 0.5269 & 0.0064 & 0.2726 & 0.8968 \\
\hline $\mathrm{HL}(\mathrm{cm})$ & $<0.0001$ & 0.0002 & 0.0410 & 0.2124 & 0.0213 & $<0.0001$ & 0.0062 \\
\hline $\mathrm{HW}(\mathrm{cm})$ & 0.0283 & 0.0092 & $<0.0001$ & 0.2161 & 0.9169 & 0.0597 & 0.0122 \\
\hline $\mathrm{SC}(\mathrm{cm})$ & 0.4564 & 0.0003 & $<0.0001$ & 0.5994 & 0.0001 & 0.6909 & 0.2794 \\
\hline HRL (cm) & $<0.0001$ & 0.0076 & 0.0007 & 0.1643 & 0.3147 & $<0.0001$ & 0.0123 \\
\hline $\mathrm{CBL}(\mathrm{cm})$ & $<0.0001$ & 0.0377 & 0.2666 & 0.6187 & 0.0349 & $<0.0001$ & 0.1417 \\
\hline $\mathrm{EL}(\mathrm{cm})$ & $<0.0001$ & 0.0365 & 0.6856 & 0.5261 & 0.4147 & 0.0002 & 0.0153 \\
\hline $\mathrm{TC}(\mathrm{cm})$ & - & - & - & 0.0253 & 0.0114 & - & - \\
\hline
\end{tabular}

$\mathrm{BL}=$ body length, $\mathrm{BW}=$ body weight, $\mathrm{HG}=$ heart girth, $\mathrm{HAW}=$ height at withers, $\mathrm{CD}=$ chest depth, $\mathrm{SPW}=$ shoulder point width, $\mathrm{SH}$ $=$ subs height, $\mathrm{RL}=$ rump length, $\mathrm{RW}=$ rump width, $\mathrm{TL}=$ tail length, $\mathrm{TW}=$ tail width, $\mathrm{HL}=$ head length, $\mathrm{HW}=$ head width, $\mathrm{SC}=$ shin circumference, $\mathrm{HRL}=$ hair length, $\mathrm{CBL}=$ canon bone length, $\mathrm{EL}=$ ear length, $\mathrm{TC}=$ testis circumference.

possessed the shortest tail and ear. Almost half of the measured traits were affected by sex of the animals showing higher values for males.

Most of the overall agro-ecological zone differences were due to the differences within the ewes. However, testis circumference, the only trait among the rams which is affected by agro-ecological zone, increases significantly as we shift from highland to lowland.

The majority of the quantitative measurements were significantly affected by the age of the animals (Figures 2, 3 and 4). Accordingly, values of some traits (body weight, chest depth, shoulder point width, rump width, testis circumference, body length, heart girth, and height at withers) gradually increased towards the optimum age of three years and then decreased towards the oldest age (5 years; Figures 2 and 3). However, this was not true in some traits (head length, head width, shin circumference, hair length, canon bone length, and ear length; Figure 4). On the other hand, age did not affect subs height, rump length, rump width, tail length, and tail width.

\section{Qualitative characteristics}

The outputs of the chi-squared tests, if the qualitative characteristics of the sheep populations from the three agro-ecological zones differ, are presented in Table 5. Accordingly, ear orientation, back profile, head profile, rump profile and coat color of the three agro-ecological zones were significantly different (Table 5). On the other hand, the coat color pattern, fiber type, skin color, tail type, tail shape and presence of toggle, horn, beard

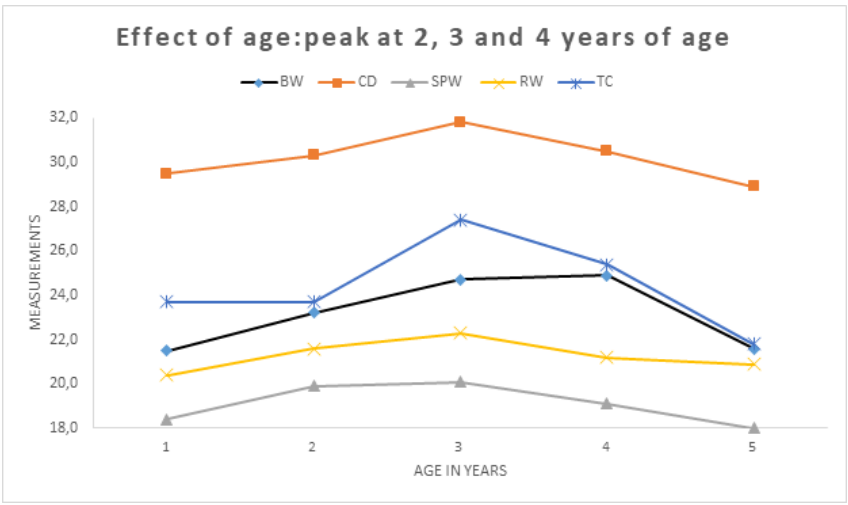

Figure 2. Effect of age on body weight(BW), chest depth (CD), shoulder point width (SPW), rump width (RW), and testis circumference (TC).

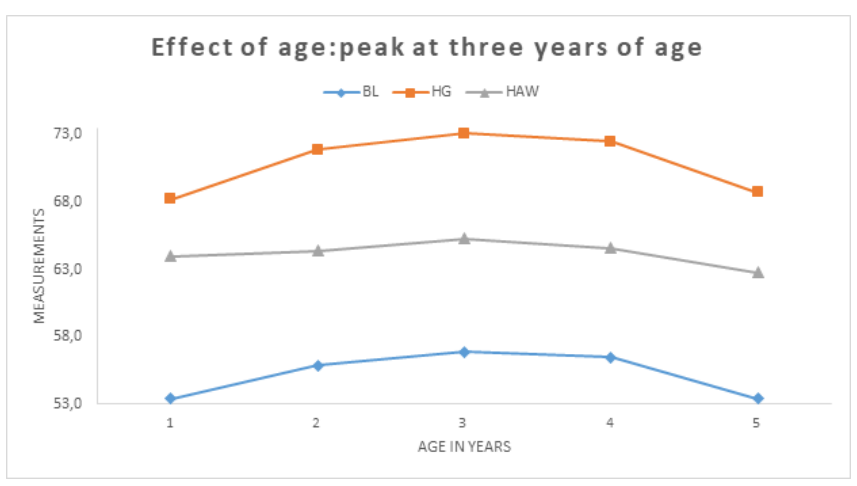

Figure 3. Effect of age on body length (BL), heart girth (HG), and height at withers (HAW) 
Table 3. Overall mean $(\overline{\mathrm{X}})$, standard error (SE), standard deviation (SD) and pairwise mean comparison (least square means and standard errors) for the effect of agro-ecological zone and sex. Means within a column bearing different superscripts are significantly different; a is given to the highest value.

\begin{tabular}{llllllll}
\hline \multirow{2}{*}{ Traits } & \multicolumn{2}{c}{ Overall } & \multicolumn{3}{c}{ Agro-ecological zone } & \multicolumn{2}{c}{ Sex } \\
& $\bar{X}_{ \pm}$SE & SD & Highland & Midland & Lowland & Rams & Ewes \\
\hline N & 306 & & 102 & 102 & 102 & 57 & 249 \\
BL (cm) & $55.2 \pm 0.2$ & 4.0 & $54.7 \pm 0.5$ & $55.4 \pm 0.4$ & $55.5 \pm 0.4$ & $55.6 \pm 0.6$ & $54.9 \pm 0.3$ \\
BW (kg) & $23.5 \pm 0.2$ & 3.4 & $22.9 \pm 0.4$ & $23.4 \pm 0.3$ & $23.3 \pm 0.4$ & $23.2 \pm 0.5$ & $23.2 \pm 0.2$ \\
HG (cm) & $71.0 \pm 0.3$ & 4.9 & $71.8 \pm 0.5^{a}$ & $70.1 \pm 0.5^{b}$ & $70.7 \pm 0.5^{a b}$ & $71.0 \pm 0.7$ & $70.7 \pm 0.3$ \\
HAW (cm) & $63.9 \pm 0.2$ & 3.2 & $63.2 \pm 0.4^{b}$ & $64.5 \pm 0.3^{a}$ & $64.9 \pm 0.4^{a}$ & $64.8 \pm 0.5$ & $63.7 \pm 0.2$ \\
CD (cm) & $29.8 \pm 0.2$ & 3.1 & $30.0 \pm 0.4$ & $30.0 \pm 0.3$ & $30.8 \pm 0.3$ & $30.9 \pm 0.5$ & $29.6 \pm 0.2$ \\
SPW (cm) & $18.7 \pm 0.1$ & 2.3 & $19.7 \pm 0.3^{a}$ & $18.6 \pm 0.3^{b}$ & $19.0 \pm 0.3^{b}$ & $19.8 \pm 0.3$ & $18.4 \pm 0.2$ \\
SH (cm) & $32.9 \pm 0.1$ & 2.5 & $32.8 \pm 0.3$ & $33.1 \pm 0.3$ & $33.1 \pm 0.3$ & $33.3 \pm 0.4$ & $32.7 \pm 0.2$ \\
RL (cm) & $14.9 \pm 0.1$ & 2.2 & $15.6 \pm 0.3^{a}$ & $15.2 \pm 0.2^{a}$ & $14.1 \pm 0.2^{b}$ & $15.2 \pm 0.3$ & $14.7 \pm 0.2$ \\
RW (cm) & $21.3 \pm 0.1$ & 2.3 & $21.2 \pm 0.3$ & $21.4 \pm 0.2$ & $21.2 \pm 0.3$ & $21.1 \pm 0.4$ & $21.4 \pm 0.2$ \\
TL (cm) & $16.8 \pm 0.2$ & 3.1 & $17.5 \pm 0.4^{a}$ & $16.3 \pm 0.3^{b}$ & $18.0 \pm 0.3^{a}$ & $18.1 \pm 0.5$ & $16.4 \pm 0.2$ \\
TW (cm) & $16.8 \pm 0.2$ & 3.0 & $18.0 \pm 0.3$ & $17.5 \pm 0.3$ & $17.5 \pm 0.3$ & $19.0 \pm 0.5$ & $16.3 \pm 0.2$ \\
HL (cm) & $13.9 \pm 0.1$ & 1.5 & $14.4 \pm 0.2^{a}$ & $14.0 \pm 0.2^{a}$ & $13.4 \pm 0.2^{b}$ & $14.2 \pm 0.2$ & $13.6 \pm 0.1$ \\
HW (cm) & $10.2 \pm 0.1$ & 1.3 & $10.4 \pm 0.1^{b}$ & $10.8 \pm 0.1^{a}$ & $10.4 \pm 0.1^{b}$ & $11.1 \pm 0.2$ & $9.9 \pm 0.1$ \\
SC (cm) & $6.8 \pm 0.1$ & 0.8 & $7.0 \pm 0.1$ & $7.1 \pm 0.1$ & $6.9 \pm 0.1$ & $7.3 \pm 0.1$ & $6.6 \pm 0.1$ \\
HRL (cm) & $4.5 \pm 0.1$ & 1.3 & $5.2 \pm 0.1^{a}$ & $4.6 \pm 0.1^{b}$ & $4.5 \pm 0.1^{b}$ & $5.2 \pm 0.2$ & $4.4 \pm 0.1$ \\
CBL (cm) & $12.3 \pm 0.1$ & 1.2 & $12.7 \pm 0.1^{a}$ & $12.5 \pm 0.1^{a}$ & $11.8 \pm 0.1^{b}$ & $12.5 \pm 0.2$ & $12.2 \pm 0.1$ \\
EL (cm) & $7.6 \pm 0.2$ & 3.0 & $7.8 \pm 0.4^{a}$ & $6.4 \pm 0.3^{b}$ & $8.4 \pm 0.4^{a}$ & $7.7 \pm 0.5$ & $7.4 \pm 0.2$ \\
\hline N $\mathrm{n}$ (cmb & & & &
\end{tabular}

$\mathrm{N}=$ number of observations, BL = body length, BW = body weight, HG = heart girth, HAW = height at withers, CD = chest depth, SPW = shoulder point width, $\mathrm{SH}=$ subs height, $\mathrm{RL}=$ rump length, $\mathrm{RW}=$ rump width, TL = tail length, TW = tail width, HL =head length, HW = head width, $\mathrm{SC}=$ shin circumference, $\mathrm{HRL}=$ hair length, $\mathrm{CBL}=$ canon bone length, EL = ear length.

Table 4. Pairwise mean comparison (least square means and standard errors) for the effect of agro-ecological zonewithin each sex. Means within a column bearing different superscripts are significantly different; a is given to the highest value.

\begin{tabular}{|c|c|c|c|c|c|c|}
\hline \multirow{2}{*}{ Traits } & \multicolumn{3}{|c|}{ Rams } & \multicolumn{3}{|c|}{ Ewes } \\
\hline & Highland & Midland & Lowland & Highland & Midland & Lowland \\
\hline $\mathrm{N}$ & 14 & 26 & 17 & 88 & 76 & 85 \\
\hline $\mathrm{BL}(\mathrm{cm})$ & $56.3 \pm 1.3$ & $56.1 \pm 0.9$ & $57.6 \pm 1.0$ & $54.7 \pm 0.5$ & $55.5 \pm 0.5$ & $55.3 \pm 0.5$ \\
\hline BW (kg) & $23.2 \pm 1.1$ & $23.7 \pm 0.7$ & $24.8 \pm 0.8$ & $23.3 \pm 0.4$ & $23.7 \pm 0.4$ & $23.3 \pm 0.4$ \\
\hline $\mathrm{HG}(\mathrm{cm})$ & $73.2 \pm 1.5$ & $70.6 \pm 1.0$ & $73.3 \pm 1.2$ & $72.2 \pm 0.6^{a}$ & $70.6 \pm 0.6^{b}$ & $70.6 \pm 0.6^{b}$ \\
\hline HAW $(\mathrm{cm})$ & $65.0 \pm 1.1$ & $65.7 \pm 0.7$ & $67.6 \pm 0.8$ & $63.1 \pm 0.4^{b}$ & $64.4 \pm 0.4^{a}$ & $64.4 \pm 0.4^{a}$ \\
\hline $\mathrm{CD}(\mathrm{cm})$ & $30.2 \pm 1.2$ & $30.8 \pm 0.8$ & $32.5 \pm 0.9$ & $29.7 \pm 0.3$ & $29.4 \pm 0.4$ & $30.2 \pm 0.4$ \\
\hline $\mathrm{SPW}(\mathrm{cm})$ & $20.0 \pm 0.9$ & $19.9 \pm 0.6$ & $20.3 \pm 0.7$ & $19.6 \pm 0.2^{a}$ & $18.1 \pm 0.3^{b}$ & $18.5 \pm 0.2^{b}$ \\
\hline $\mathrm{SH}(\mathrm{cm})$ & $32.0 \pm 1.0$ & $34.0 \pm 0.6$ & $33.3 \pm 0.7$ & $32.7 \pm 0.3$ & $32.7 \pm 0.3$ & $32.8 \pm 0.3$ \\
\hline $\mathrm{RL}(\mathrm{cm})$ & $15.3 \pm 0.8$ & $14.8 \pm 0.5$ & $14.9 \pm 0.6$ & $15.5 \pm 0.3^{a}$ & $15.2 \pm 0.3^{a}$ & $13.7 \pm 0.3^{b}$ \\
\hline $\mathrm{RW}(\mathrm{cm})$ & $22.6 \pm 0.8$ & $21.4 \pm 0.5$ & $22.1 \pm 0.6$ & $21.6 \pm 0.3$ & $21.9 \pm 0.3$ & $21.4 \pm 0.3$ \\
\hline TL $(\mathrm{cm})$ & $19.2 \pm 1.2$ & $17.2 \pm 0.8$ & $18.7 \pm 0.9$ & $16.9 \pm 0.3^{a}$ & $15.7 \pm 0.4^{b}$ & $17.4 \pm 0.3^{a}$ \\
\hline TW $(\mathrm{cm})$ & $19.9 \pm 1.1$ & $18.7 \pm 0.7$ & $19.3 \pm 0.8$ & $16.9 \pm 0.3$ & $16.4 \pm 0.4$ & $16.3 \pm 0.3$ \\
\hline $\mathrm{HL}(\mathrm{cm})$ & $15.2 \pm 0.6$ & $14.6 \pm 0.4$ & $14.1 \pm 0.4$ & $14.2 \pm 0.2^{a}$ & $13.8 \pm 0.2^{a}$ & $13.2 \pm 0.2^{b}$ \\
\hline $\mathrm{HW}(\mathrm{cm})$ & $10.4 \pm 0.6$ & $11.4 \pm 0.4$ & $11.2 \pm 0.5$ & $9.8 \pm 0.1$ & $10.2 \pm 0.1$ & $9.7 \pm 0.1$ \\
\hline $\mathrm{SC}(\mathrm{cm})$ & $7.4 \pm 0.3$ & $7.7 \pm 0.2$ & $7.5 \pm 0.3$ & $6.7 \pm 0.1$ & $6.7 \pm 0.1$ & $6.6 \pm 0.1$ \\
\hline HRL $(\mathrm{cm})$ & $4.9 \pm 0.5$ & $5.4 \pm 0.3$ & $4.5 \pm 0.4$ & $5.0 \pm 0.1^{a}$ & $4.1 \pm 0.2^{b}$ & $4.2 \pm 0.1^{b}$ \\
\hline $\mathrm{CBL}(\mathrm{cm})$ & $12.6 \pm 0.4$ & $12.9 \pm 0.2$ & $12.6 \pm 0.3$ & $12.8 \pm 0.1^{a}$ & $12.4 \pm 0.1^{a}$ & $11.7 \pm 0.1^{b}$ \\
\hline $\mathrm{EL}(\mathrm{cm})$ & $7.6 \pm 1.1$ & $7.2 \pm 0.7$ & $8.3 \pm 0.8$ & $7.5 \pm 0.4^{a}$ & $6.1 \pm 0.4^{b}$ & $8.2 \pm 0.4^{a}$ \\
\hline $\mathrm{TC}(\mathrm{cm})$ & $23.2 \pm 1.1^{b}$ & $24.0 \pm 0.7^{a b}$ & $26.1 \pm 0.8^{a}$ & - & - & - \\
\hline
\end{tabular}

$\mathrm{N}=$ number of observations, BL = body length, BW = body weight, HG = heart girth, HAW = height at withers, CD = chest depth, SPW = shoulder point width, $\mathrm{SH}=$ subs height, $\mathrm{RL}=$ rump length, $\mathrm{RW}=$ rump width, $\mathrm{TL}=$ tail length, $\mathrm{TW}=\mathrm{tail}$ width, $\mathrm{HL}=$ head length, $\mathrm{HW}=\mathrm{head}$ width, $\mathrm{SC}=$ shin circumference, $\mathrm{HRL}=$ hair length, $\mathrm{CBL}=$ canon bone length, EL = ear length, TC = testis circumference. 


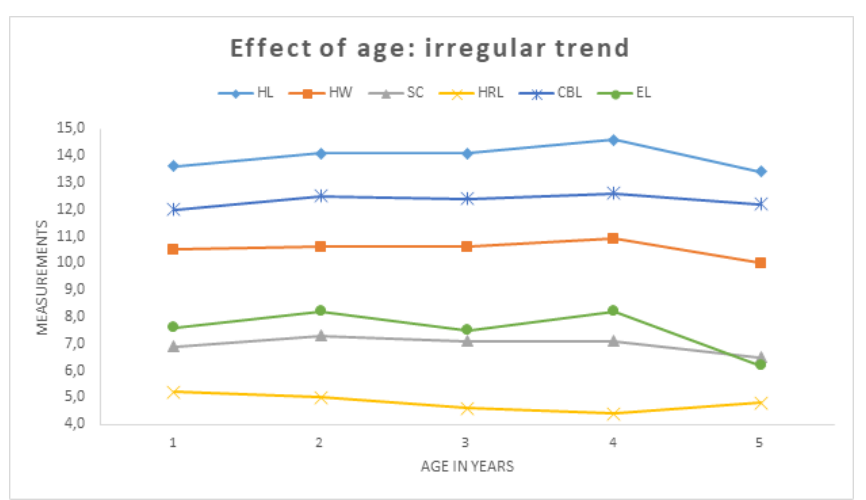

Figure 4. Effect of age on head length(HL), head width (HW), shin circumference (SC), hair length (HRL), canon bone length (CBL), and ear length (EL)

and ruff were not significantly different among the studied agro-ecological zones. Accordingly, the sheep populations can be characterized as hair type sheep with plain coat color pattern. Additionally, all of the studied sheep populations possess cylindrical thin tail with turned up at end, and straight head profile. The results also revealed that almost none of the sheep sampled have pigmented skin, horns, toggle, ruff and beard. Tan coat color was dominantly observed in the high and midland agro-ecological zones while white and brown colors were dominant in the lowland sheep (Figures 5, 6 and 7).

\section{Multivariate analysis for discrimination of sheep populations}

According to stepwise discriminant function analysis, canon bone length, height at withers and tail length were the three most important morphometric variables used in discriminating the sheep populations from different agro-ecological zones (Table 6). Chest depth, subs height, body length, and tail width were found not to be useful variables due to their lowest discriminatory power (Table 6).

The probabilities of all main multivariate tests over the canonical structures were significant (Table 7).

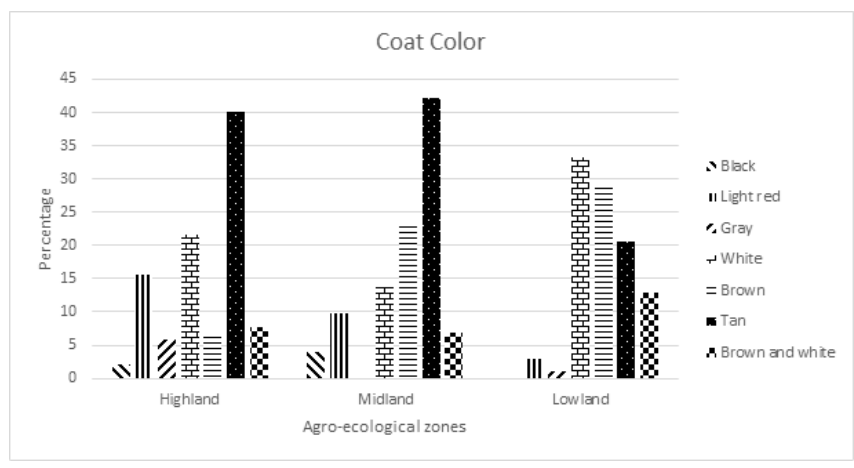

Figure 5. Coat color of sheep populations from different agro-ecological zones; significant $(\mathrm{p}<0.0001)$ effect of agroecological zones was observed over the coat color of the studied sheep populations.
Table 5. Percentages and their respective chi-squared probabilities of some qualitative characteristics of the sheep populations from different agro-ecological zones. $\chi^{2}$ values in bold are statistically significant.

\begin{tabular}{|c|c|c|c|c|}
\hline \multirow{2}{*}{$\begin{array}{l}\text { Qualitative } \\
\text { traits }\end{array}$} & \multicolumn{3}{|c|}{ Agro-ecological zones } & \multirow[b]{2}{*}{$\chi^{2}$} \\
\hline & Highland & Midland & Lowland & \\
\hline $\begin{array}{l}\text { Coat color } \\
\text { pattern }\end{array}$ & & & & 0.1445 \\
\hline Plain & 77.5 & 85.3 & 82.4 & \\
\hline Patchy & 17.6 & 12.7 & 17.6 & \\
\hline Spotted & 4.9 & 2.0 & NR & \\
\hline Fiber type & & & & 0.8211 \\
\hline Hairy & 85.3 & 86.3 & 88.2 & \\
\hline Wooly & 14.7 & 13.7 & 11.8 & \\
\hline $\begin{array}{l}\text { Ear } \\
\text { orientation }\end{array}$ & & & & 0.0127 \\
\hline Erect & 1.0 & 1.0 & 4.9 & \\
\hline $\begin{array}{l}\text { Semi- } \\
\text { pendulous }\end{array}$ & 48.0 & 34.3 & 37.2 & \\
\hline Pendulous & 25.5 & 16.7 & 21.6 & \\
\hline $\begin{array}{l}\text { Carried } \\
\text { horizontal }\end{array}$ & 25.5 & 48.0 & 36.3 & \\
\hline Head profile & & & & 0.0100 \\
\hline Straight & 71.6 & 65.7 & 85.3 & \\
\hline Concave & 12.7 & 11.8 & 8.8 & \\
\hline Convex & 15.7 & 22.5 & 5.9 & \\
\hline Back profile & & & & 0.0002 \\
\hline Straight & 70.5 & 40.2 & 62.8 & \\
\hline $\begin{array}{l}\text { Slopes up } \\
\text { towards the } \\
\text { rump }\end{array}$ & 25.5 & 56.9 & 34.3 & \\
\hline $\begin{array}{l}\text { Slopes down } \\
\text { from withers }\end{array}$ & 2.0 & 2.9 & 2.9 & \\
\hline Curved & 2.0 & 0 & 0 & \\
\hline Rump profile & & & & $<0.000$ \\
\hline Flat & 27.5 & 59.8 & 50.0 & \\
\hline Sloping & 72.5 & 40.2 & 48.0 & \\
\hline Roofy & 0 & 0 & 2.0 & \\
\hline Toggle & & & & 0.0987 \\
\hline Present & 3.9 & 11.8 & 10.8 & \\
\hline Absent & 96.1 & 88.2 & 89.2 & \\
\hline
\end{tabular}

Canonical correlation coefficients of the quantitative variables and class means outputs from the two canonical structures are shown in Table 8 . The first canonical structure (Can 1) explains the majority (69\%) of the variability with eigenvalue of 0.48 . The first canonical correlation (57\%) was the greatest multiple correlation with the classes that was achieved by using the linear combination of the quantitative variables. The results revealed that Can 1 separates the sheep populations (class means) from different agro-ecological zones.

Results of a discriminant function analysis (Table 9) shows the classification of data into a known agroecological zone. Accordingly, an average of $66 \%$ of the sampled animals were classified into their respective 
Table 6. Summary of the stepwise discriminant function analysis; ascending order of traits used in discriminating the sheep populations from different agro-ecological zones.

\begin{tabular}{lllllll}
\hline Step & Variables entered & Partial R-squared & F value & Pr $>$ F & Wilk's Lambda & Pr $<$ Lambda \\
\hline 1 & Canon bone length & 0.1237 & 21.39 & $<0.0001$ & 0.8763 & $<0.0001$ \\
2 & Height at withers & 0.0564 & 9.02 & 0.0002 & 0.8269 & $<0.0001$ \\
3 & Tail length & 0.0557 & 8.87 & 0.0002 & 0.7809 & $<0.0001$ \\
4 & Rump length & 0.0554 & 8.79 & 0.0002 & 0.7376 & $<0.0001$ \\
5 & Head width & 0.0529 & 8.36 & 0.0003 & 0.6986 & $<0.0001$ \\
6 & Hair length & 0.0460 & 7.18 & 0.0009 & 0.6664 & $<0.0001$ \\
7 & Head length & 0.0525 & 8.23 & 0.0003 & 0.6315 & $<0.0001$ \\
8 & Shoulder point width & 0.0308 & 4.70 & 0.0098 & 0.6120 & $<0.0001$ \\
9 & Shin circumference & 0.0258 & 3.91 & 0.0210 & 0.5962 & $<0.0001$ \\
10 & Heart girth & 0.0172 & 2.58 & 0.0777 & 0.5860 & $<0.0001$ \\
11 & Body weight & 0.0268 & 4.03 & 0.0188 & 0.5703 & $<0.0001$ \\
12 & Rump width & 0.0147 & 2.18 & 0.1154 & 0.5619 & $<0.0001$ \\
- & Chest depth & 0.0073 & 1.07 & 0.3432 & - & - \\
- & Subs height & 0.0013 & 0.18 & 0.8314 & - & - \\
- & Body length & 0.0010 & 0.15 & 0.8595 & - & - \\
- & Tail width & 0.0008 & 0.12 & 0.8876 & - & - \\
\hline
\end{tabular}

agro-ecological zone. The overall error rate was $34 \%$, while higher error rates were obtained from the classification of midland sheep populations.

Pairwise squared distances between agro-ecological zones are shown in Table 10. All distances were significant. Highland sheep populations are distantly related to the lowland sheep. On the other hand, midland sheep relates more towards lowland sheep than highland sheep.

Table 7. Multivariate statistics and F approximates

\begin{tabular}{llllll}
\hline Statistic & Value & $\begin{array}{l}\mathrm{F} \\
\text { Value }\end{array}$ & $\begin{array}{l}\text { Num } \\
\text { DF }\end{array}$ & $\begin{array}{l}\text { Den } \\
\text { DF }\end{array}$ & Pr $>$ F \\
\hline $\begin{array}{l}\text { Wilks' } \\
\text { Lambda }\end{array}$ & 0.5559 & 6.14 & 32 & 576 & $<0.0001$ \\
$\begin{array}{l}\text { Pillai's Trace } \\
\text { Hotelling- }\end{array}$ & 0.5015 & 6.04 & 32 & 578 & $<0.0001$ \\
$\begin{array}{l}\text { Lawley } \\
\text { Trace }\end{array}$ & 0.6957 & 6.24 & 32 & 510.82 & $<0.0001$ \\
$\begin{array}{l}\text { Roy's } \\
\text { Greatest Root }\end{array}$ & 0.4811 & 8.69 & 16 & 289 & $<0.0001$ \\
\hline
\end{tabular}

Table 8. Eigen values, canonical correlations and class means

\begin{tabular}{lll}
\hline & Can 1 & Can 2 \\
\hline Multivariate Statistics & & \\
Canonical correlation & $\mathbf{0 . 5 6 9 9}$ & 0.4203 \\
Eigenvalue & $\mathbf{0 . 4 8 1 1}$ & 0.2146 \\
Proportion & $\mathbf{0 . 6 9 1 6}$ & 0.3084 \\
Class (agro-ecological zones) & & \\
Highland & $\mathbf{0 . 9 1 3 9}$ & -0.2290 \\
Lowland & $\mathbf{- 0 . 7 5 3 9}$ & -0.4141 \\
Midland & $\mathbf{- 0 . 1 6 0 0}$ & 0.6430 \\
\hline
\end{tabular}

\section{Discussion}

Along with the most observable qualitative traits, quantitative measurements produce reliable information in characterization and differentiation of sheep populations. In our current study, more traits showed significant differences among ewes than among rams in different agro-ecological zones. This might be due to either the larger sample size taken for ewe populations or due to the similarity of rams over the studied agro-ecological zones, which could be attributed to common markets

Table 9. Number and percent of observations classified into agro-ecological zones.

\begin{tabular}{lllll}
\hline From & Highland & Lowland & Midland & Total \\
\hline $\begin{array}{l}\text { High- } \\
\text { land }\end{array}$ & $70(68.6)$ & $20(19.6)$ & $\begin{array}{l}12 \\
(11.8)\end{array}$ & $\begin{array}{l}102 \\
(100)\end{array}$ \\
Lowland & $12(11.8)$ & $71(69.6)$ & $\begin{array}{l}19 \\
(18.6)\end{array}$ & $\begin{array}{l}102 \\
(100)\end{array}$ \\
Midland & $19(18.6)$ & $23(22.6)$ & $\begin{array}{l}60 \\
(58.8)\end{array}$ & $\begin{array}{l}102 \\
(100)\end{array}$ \\
Total & 101 & 114 & 91 & 306 \\
& $(33.0)$ & $(37.3)$ & $(29.7)$ & $(100)$ \\
$\begin{array}{l}\text { Error } \\
\text { rate }\end{array}$ & 0.31 & 0.30 & 0.41 & 0.34 \\
Priors & 0.33 & 0.33 & 0.33 & \\
\hline
\end{tabular}

Table 10. Squared Mahalanobis distance between agroecological zones; output of the multivariate analysis calculated using the quantitative measurements

\begin{tabular}{llll}
\hline From & Highland & Lowland & Midland \\
\hline Highland & 0 & \\
Lowland & $2.82^{* * *}$ & 0 \\
Midland & $1.91^{* * *}$ & $1.47^{* * *}$ & 0 \\
\hline$* * *$ shows the significance of the distance calcula- \\
tions at $\mathrm{p}<0.0001$.
\end{tabular}




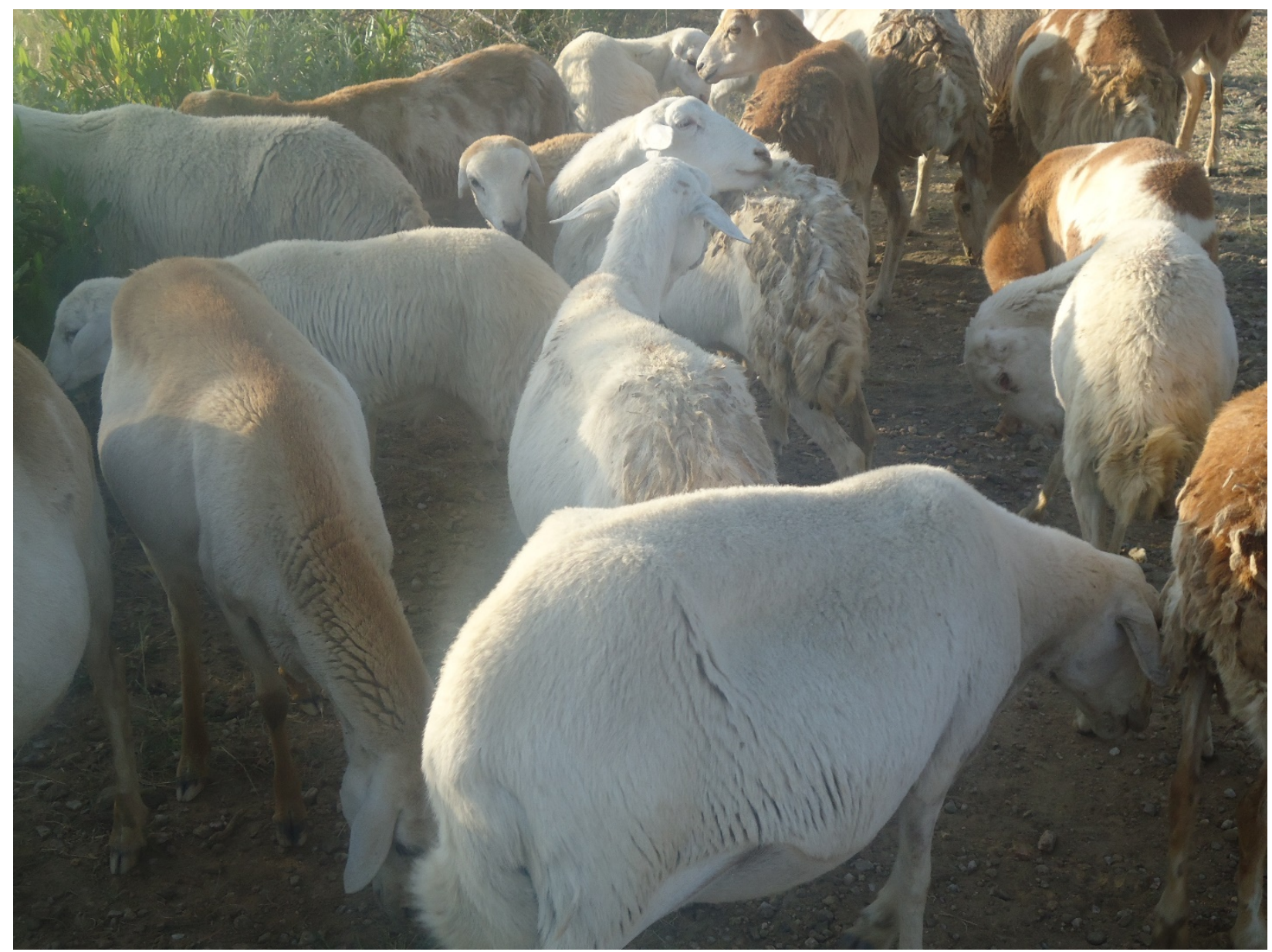

Figure 6. Lowland sheep flock in Tahtay Maichew district.

where farmers select and purchase rams for sire purposes.

Most of the studied traits were affected by agroecological zone differences, which might be due to the differences in physiological adaptation mechanism of sheep types to different environments, management, availability of different feed and nutrition and/or the variations being caused by genetic factors. For example, the majority of lowland sheep possess higher values for height at withers, testis circumference and short hair which might help them to adapt to a hot environment. These results are in line with the results of Getachew et al (2009) and Gizaw et al (2008) for Menz and Afar sheep who reported that such measurements of the lowland Afar sheep were higher than the highland Menz sheep populations.

Most of the body measurements were higher for rams than ewes, which might be attributed to enhanced muscle mass and skeletal development in males due to testosterone hormone secretions (Baneh and Hafezian, 2009). These results follow Rensch's rule where the males of a particular species are usually heavier than the females (Rensch, 1950). Size differences may be ascribed to the differences in the endocrine system of the two sexes; estrogen hormone was shown to have a limited effect on growth in females (Baneh and Hafezian, 2009). These results are in agreement with Mustefa et al (2019) and Getachew et al (2009) who reported that males were higher than the females in most growth traits in goats and sheep respectively.

In agreement with the results of the current study, most scholars report differences in traits between the sexes with rams being dominant over ewes (Rensch, 1950; Baneh and Hafezian, 2009; Mustefa et al, 2019; Getachew et al, 2009). However, differences due to sex was not observed in Tigray Highland sheep populations (Hayelom et al, 2014). In contrast to this, Hayelom et al (2014) report the dominance of ewes over the rams on most of the linear body measurements of Elle sheep populations. In a different study, no significant differences were observed among body weight of Simien sheep ewes and rams (Melaku et al, 2019).

The overall mean body weight $(23.50 \mathrm{~kg})$ presented in the current study was higher than those reported by Tajebe et al (2011) for Abergelle sheep (21.25 kg), and by Gebreyowhens and Tesfay (2016) for Tigray Highland sheep (22.10 kg), while the values were lower than those reported by Hayelom et al (2014) for Tigray Highland sheep (27.52 kg), and Melaku et al (2019) for Simien sheep $(24.90 \mathrm{~kg})$ which show their difference from the sheep populations of the neighboring areas. Although the body weight of rams and ewes presented in this study were higher than those reported for 


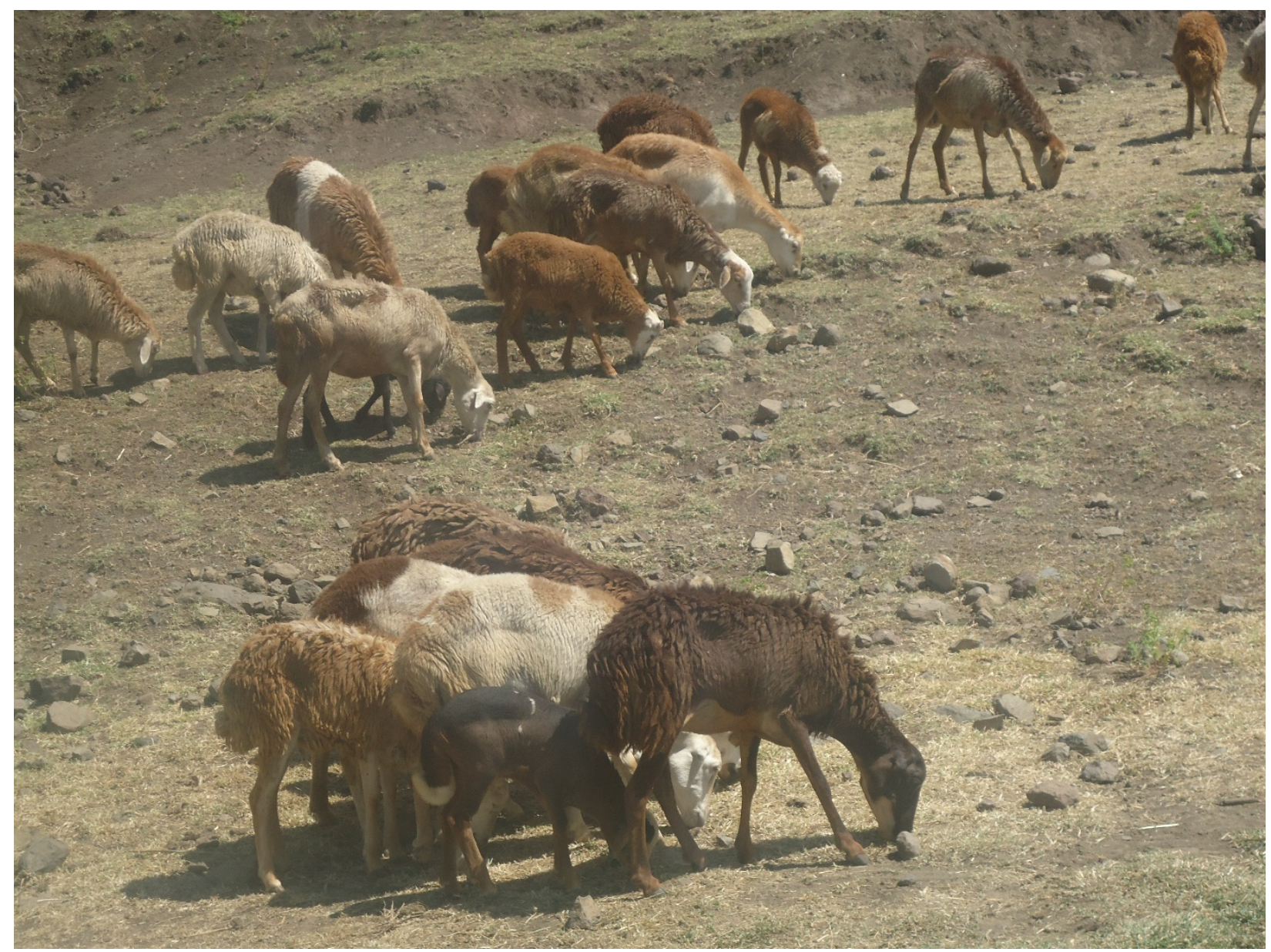

Figure 7. Highland sheep flock in Tahtay Maichew district.

Abergelle and Tigray highland sheep (Tajebe et al, 2011; Gebreyowhens and Tesfay, 2016), other traits such as body length, height at withers, heart girth and tail length were comparable between these sheep populations. On the other hand, higher values for all morphological measurements were reported by Edea et al (2010) for the country's most known sheep breeds (Bonga and Horro sheep).

The results also revealed that linear body measurements among the studied sheep population differ with age. Three-year-old sheep showed the highest values for most of the measurements, reflecting the optimum growth age. These results are in contrast with results of Getachew et al (2009) for Menz and Afar sheep and Melaku et al (2019) for Simien sheep who reported that the body weight of the sheep continued to increase with age.

In addition to the quantitative measurements, the qualitative characteristics of a population also allow to easily differentiate genetic resources. Among the obvious qualitative characteristics which differentiate the current sheep populations from the previously characterized sheep populations are the complete absence of beard, horn, ruff, toggle and pigmented skin. Similarly, variations in coat color were also observed among the different agro-ecological zones. Accordingly, the majority of the highland and midland sheep populations from the current study possess tan coat color which makes them unique among the other Tigray highland sheep populations. Sheep populations sampled from the lowland area display dominantly white and light colors which is in agreement with the report of Getachew et al (2009) for the lowland Afar sheep. The majority of the sheep populations from the current study possessed hairy fiber type coats, which was in contrast to the results of Hayelom et al (2014), who report course wool for Tigray Highland sheep. However, huge variations were not observed among the other qualitative characteristics of the sheep types from the current study and earlier studies of Gebreyowhens and Tesfay (2016) and Hayelom et al (2014) for Tigray Highland sheep.

Discriminant function analysis allowed the classification of an average $66 \%$ of the studied animals into their respective environments zone. Lowest classification of individuals into their respective agro-ecological zone was observed in midland sheep populations, indicating a lack of uniqueness within them. All of the pairwise comparisons between populations from different agro-ecological zones were found to be highly significant with the largest difference observed between the highland and lowland sheep populations. These results reflect the large altitudinal differences between the two agro-ecological zones. The shortest distance calculated 
between the lowland and midland sheep populations show their relative similarities as compared with the highland sheep. These differences among different agroecological zones show the presence of potential genetic resource variations which can be useful for maintaining diversity and further selection-based genetic improvement programs.

In conclusion, using a combination of quantitative and qualitative characteristics we were able to discriminate the sheep populations from three agro-ecological zones in the Tahtay Maichew district, Tigray region of Ethiopia and to group them into two distinct populations (the highland and the lowland sheep). Sheep populations from the midland agro-ecological zone were considered to be part of the lowland group. Therefore, it is better to consider the highland and lowland sheep as different traditional populations until molecular characterization results provide further evidence for population differentiation. Additionally, the molecular characterization studies will show the within population genetic diversity and level of inbreeding which can be used for selecting appropriate genetic improvement plans (selection or crossbreeding). According to the reports of Gizaw et al (2008), the sheep genetic resources of most parts of the Tigray region were generally referred as Sekota sheep population under the short fat tailed breed. However, results from our study indicate that there are several sheep populations that cannot be categorized under the Sekota traditional sheep population. Therefore, it is advisable to include these genetic resources for further molecular studies to understand the genetic diversity within and among populations.

\section{Acknowledgements}

The authors are highly indebted to the Ethiopian Biodiversity Institute (EBI) for covering all the budget needs of the work. Our special appreciation also goes to the smallholder farmers /breeders for providing their animal to this work for free. We also take this opportunity to thank the animal science experts and development agents in the district for their endless help during the data collection. A special word also goes to our friend and work partner Mr. Tadesse Hunduma for mapping the study area.

\section{Author contributions}

All authors contributed to the study conception and design. Material preparation, and data collection were performed by AH, TA, SS and ST. Data analysis and writing the first draft of the manuscript was performed by $\mathrm{AM}$ and $\mathrm{AH}$. AA commented on previous versions of the manuscript. All authors read and approved the final manuscript.

\section{Conflict of interest statement}

The authors declare no conflicts of interest.

\section{References}

Atsbeha, G., Demissew, S., Woldu, Z., and Edwards, S. (2015). Floristic composition of herbaceous flowering plant species in Lalay and Tahtay Michew Districts, Central Zone of Tigray, Ethiopia. African Journal of Ecology and Ecosystems 2(6), 159-169.

Baneh, H. and Hafezian, S. H. (2009). Effect of environmental factor on growth traits in Ghezel sheep. African Journal of Biotechnology 8, 2903-2907. url: https://www.ajol.info/index.php/ajb/article/view/ 60943.

Bimerow, T., Yitayew, A., Taye, M., and Mekuriaw, S. (2011). Morphological Characteristics of Farta Sheep in Amhara Region, Ethiopia. Online Journal of Animal and Feed Research 1(6), 299-305.

Central Statistical Agency (2005). Ethiopian agricultural sample survey 2012/2013 (2005 E.C.). In Report on livestock and livestock characteristics., volume II, Central Statistical Agency, Addis Ababa, Ethiopia.

Central Statistical Agency (2018). Ethiopian agricultural sample survey 2017/18 (2010 E.C.). In Report on livestock and livestock characteristics, volume II, Central Statistical Agency, Addis Ababa, Ethiopia.

Degen, A. A. (2007). Sheep and goat milk in pastoral societies. Small Ruminant Research 68(1-2), 7-19. url: https://dx.doi.org/10.1016/j.smallrumres.2006. 09.020. doi: 10.1016/j.smallrumres.2006.09.020

Devendra, C. and Mcleroy, G. B. (1982). Goat and sheep production in the tropics Intermediate tropical agriculture series, (London: Longman), 271p.

EBI (2016). Ethiopian National Strategy and Plan of Action for conservation and utilization of Animal Genetic Resources (Addis Ababa, Ethiopia: Ethiopian Biodiversity Institute), 114-114. url: https://www.ebi.gov.et/wp-content/uploads/2013/ 01/Final\%20ENSAP final submitted\%20(1).pdf.

Edea, Z., Haile, A., Tibbo, M., Sharma, A. K., Assefa, D., Sölkner, J., and Wurzinger, M. (2010). Morphological characterization of Bonga and Horro indigenous sheep breeds under smallholder conditions in Ethiopia. Ethiopian Journal of Animal Production 9(1), 117133.

FAO (2012). Phenotypic characterization of Animal Genetic Resources. url: www.fao.org/docrep/015/ i2686e/i2686e00.pdf.

Gebreyowhens, W. and Tesfay, Y. (2016). Morphological Characterization of Indigenous Highland Sheep Population of Tigray, Northern Ethiopia. Journal of Natural Sciences Research 6(1), 96104. url: https://www.iiste.org/Journals/index.php/ JNSR/article/view/28281/0.

Getachew, T., Haile, A., Tibbo, M., Sharma, A. K., Kifle, A., Terefe, E., Wurzinger, M., and Sölkner, J. (2009). Morphological characters and body weight of Menz and Afar sheep within their production system. Ethiopian Journal of Animal Production 9(1), 99-115.

Gizaw, S., Komen, H., Hanotte, O., and Arendonk, J. A. M. V. (2008). Indigenous sheep resources of Ethiopia: types, production systems and farmers preferences. 
Animal Genetic Resources Information 43, 25-39. url: https://dx.doi.org/10.1017/s1014233900002704. doi: $10.1017 / \mathrm{s} 1014233900002704$

Hayelom, M., Abegaz, S., and Mekasha, Y. (2014). Within Breed Phenotypic Diversity of Sokota/Tigray Sheep in Three Selected Zones of Tigray, Northern Ethiopia. Journal of Biology Agriculture and Healthcare 4(17), 148-157. url: https://www.iiste.org/Journals/ index.php/JBAH/article/view/15182.

Melaku, S., Kidane, A., Abegaz, S., Tarekegn, A., and Tesfa, A. (2019). Phenotypic characterization of Simien sheep in Simien Mountain Region. Ethiopia. International Journal of Agriculture and Biosciences 8(4), 178-185. url: http://www.ijagbio.com/volume8-no-4-2019/.

Mengistie, T., Girma, A., Solomon, G., Sisay, L., Abebe, M., and Markos, T. (2010). Traditional management systems and linear body measurements of Washera sheep in the western highlands of the Amhara National Regional State, Ethiopia. Livestock Research for Rural Development 22(169). url: http://www.lrrd. org/lrrd22/9/taye22169.htm.

Michael, A., Kefelegn, K., and Yoseph, M. (2016). Phenotypic Characterization of Indigenous Sheep Types in Northern Ethiopia. Journal of Natural Sciences Research 6(15), 16-27. url: https://iiste.org/Journals/index.php/JNSR/article/ view/32220/33107.

Misra, A. K. and Singh, K. (2002). Effect of water deprivation on dry matter intake, nutrient utilization and metabolic water production in goats under semiarid zones of India. Small Ruminant Research 46, 159165. doi: https://doi.org/10.1016/S0921-4488(02) 00187-6

Mustefa, A., Gizaw, S., Banerjee, S., Abebe, A., Taye, M., Areaya, A., and Besufekad, S. (2019). Growth performance of Boer goats and their F1 and F2 crosses and backcrosses with Central Highland goats in Ethiopia. Livestock Research for Rural Development 31(6), 89. url: http://www.lrrd.org/ lrrd31/6/amine31089.html.

Rensch, B. (1950). Die Abhängigkeit der relativen Sexualdifferenz von der Körpergröße. Bonner Zoologische Beitrage 1, 58-69.

SAS Institute (2002). Statistical Analysis Software (SAS) url: https://www.sas.com/en_us/software/stat.html.

Tajebe, S., Gangwar, S. K., and Kebede, K. (2011). Performance and Physical Body Measurement of Abergele Sheep Breed in Traditional Management System of Tigray Regional State, Northern Ethiopia. International Journal of Science and Nature 2(2), 225230. url: https://hdl.handle.net/10568/70955.

Worku, A. (2018). Morphometrical Characterization and Traditional Breeding Objectives of Native Sheep Reared in East Shoa and West Arsi Zone of Oromia Regional State. M.Sc. thesis, Hawassa University, Hawassa, Ethiopia. 